\title{
Variability in the prevalence of premenstrual asthma
}

\author{
A. Pereira Vega*, J.L. Sánchez Ramos ${ }^{\#}$, J.A. Maldonado Pérez*, F.J. Álvarez Gutierrez", \\ J.M. Ignacio García ${ }^{+}$, R. Vázquez Oliva ${ }^{\S}$, P. Romero Palacios ${ }^{\dagger}$, J.M. Bravo Nieto*, \\ I. Sánchez Rodríguez* and F. Gil Muñoz*
}

ABSTRACT: Our aim is to analyse the differences in the prevalence of premenstrual asthma (PMA) according to a set of criteria, the relationship between them and the influence of asthma severity.

The answer "Yes" to "Does your asthma get worse before menstruation?" was considered subjective PMA. A daily respiratory symptoms register of fertile asthmatic females was taken during two consecutive menstrual cycles. For the semi-objective diagnosis, an exacerbation of $\geqslant 20 \%$ was required in the symptoms register. Objective diagnosis was a premenstrual worsening of $\geqslant 20 \%$ of peak flow.

We selected 103 patients. Subjective premenstrual deterioration was perceived in $43.7 \%$. The semi-objective deterioration of symptoms in the first cycle occurred in $44.7 \%$, and in $22.3 \%$ in both cycles. A total of $54.3 \%$ of females with semi-objective criteria in the first cycle perceived a subjective deterioration of symptoms, versus $35.1 \%$ of those without semi-objective criteria $(p=0.05)$. PMA was present at all levels of asthma severity, with no clear link to the degree of severity.

The detection of PMA prevalence, the subjective perception of this deterioration and its presence at all levels of asthma severity lead us to urge research into possible premenstrual deterioration in all fertile asthmatic females.

\section{KEYWORDS: Menstrual cycle, premenstrual asthma, prevalence}

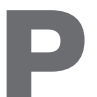

remenstrual asthma (PMA) is considered to be the cyclical deterioration of the asthmatic condition during the premenstrual phase and/or the first days of menstruation. The exacerbation of the respiratory symptoms and/or peak flow (PF) deterioration affects $>30 \%$ of asthmatic females, according to various studies $[1,2]$. The most notable asthmatic symptoms evaluated are coughing, wheezing, tightness across the chest and breathlessness [1-4].

The definition of PMA varies according to different studies. While some authors require only the patient's subjective manifestation of the premenstrual deterioration of the asthmatic symptoms, others need a methodology with a series of objective criteria that show premenstrual deterioration of the symptoms or of functional parameters such as PF values [1, 4]. Likewise, while some studies require these criteria to be satisfied in one cycle [4], others deem it necessary for them to occur in two consecutive cycles [5].
Three groups of patients have been observed in wide-ranging studies of asthmatic females of fertile age [6]: patients who suffered deterioration neither of their symptoms nor of PF; others who had a slight exacerbation of symptoms and reduction of PF values, controlling their symptoms with an increased dose of their normal medication; and those who suffered a clear deterioration in their asthma and/or a significant exacerbation of PF values, their asthma being difficult to control, occasionally requiring admittance to hospital or an emergency department.

This latter group accounts for $4 \%$ of the total number of asthmatics of fertile age [6], and is the most clinically relevant, the most studied and possibly the reason why PMA is deemed to be asthma that is serious, unstable and hard to control.

We are unaware of any study that analyses the prevalence of PMA in Spain or its distribution among the various groups of asthma severity according to the Global Initiative for Asthma (GINA) [7]. Besides, as TAN [6] points out,

\section{AFFILIATIONS}

*Pneumology Section, Hospital Juan Ramón Jiménez de Huelva, ${ }^{\#}$ Dept of Nursing, University of Huelva, Campus del Carmen, ${ }^{\S}$ Pneumology Section, Hospital Infanta Elena, Huelva,

-Pneumology Service, Hospital Virgen del Rocío, Seville, +Pneumology Section, Hospital de la Serranía de Ronda, Málaga, and fPneumology Section, Hospital de Baza, Granada, Spain.

CORRESPONDENCE

A. Pereira Vega

C/Puerto 32

$7^{\circ}$ izquierda

Huelva 21001

Spain

E-mail: apv01h@saludalia.com

Received:

March 192009

Accepted after revision:

Oct 122009

First published online:

Nov 062009 
large-scale community studies are needed to determine the true extent of prevalence.

The aim of our study is to analyse the differences in the prevalence of PMA according to a set of subjective, semiobjective and objective criteria, the relationship between these sets of criteria and the influence of the severity of asthma.

\section{MATERIAL AND METHODS}

The study was carried out in the outpatients' clinics of five hospitals in Andalusia (Spain). It looked at asthmatic females of fertile age. Asthma was determined by a doctor's diagnosis based on the clinical and reversible deterioration of $>15 \%$ in $\mathrm{PF}$ or spirometry. Criteria for exclusion were pregnancy or lactation.

Informed consent was sought after explaining the purpose of the study. Data were gathered on the patient's indication of the existence of an exacerbation of asthma and its relationship with the menstrual cycle, whether the cycles were regular (from 47 days every $28 \pm 5$ days), the use of anti-asthma medication in the previous 2 months, as well as the possible use of oral contraceptives (OC).

PMA was considered from the subjective viewpoint when "Yes" was the answer to the question "Does your asthma get worse before menstruation?"

To define PMA from a semi-objective viewpoint, we applied the methodology used in studies by ELIASSON et al. [1] and ENSOM et al. [4]. The study involved drawing up a clinical history protocol and the completion of symptoms questionnaires gathered daily in two consecutive menstrual cycles. The symptoms questionnaire recorded the presence of coughing, wheezing, tightness across the chest and breathlessness. The daily presence of the symptoms studied was catalogued from zero to three $[4,8]$. Zero defined the absence of symptoms, one referred to mild symptoms (slight interference with normal activity), two was for moderate symptoms (interference with normal activity without impeding work or school attendance) and three indicated severe symptoms (interference with normal activity leading to absence at work, school or cancellation of appointments). Data on the anti-asthma medication taken was also collected.

Data interpretation was according to the following steps: 1) daily evaluation (zero to three) of the four symptoms questionnaires gathered; 2) daily score index; 3) average of score indexes over two 6-day periods, from the fifth to the 10th day (the first day being the start of menstruation), which would correspond to the follicular or preovulatory phase, and the last 5 days of the cycle, including the first day of menstruation, corresponding to the luteal phase; 4) the difference between these two values, which was significant if $>20 \%$. Using the information on symptoms from the questionnaire, and taking into account the frequency of symptoms from the GINA scale of severity [7], our patients were then distributed among the various groups according to severity. PMA is considered semi-objective if premenstrual deterioration is $>20 \%$ of symptoms. This would need to be so in one or two of the menstrual cycles $[4,9]$.

We used PF to define objective PMA criteria. For PF, measurements were taken twice daily in the first menstrual cycle, in the morning and evening, with the best of the three measurements from each session selected. The average of morning and evening values was obtained in the two phases analysed (preovulation and premenstruation), calculating the percentage difference between these two values. If the difference was $\geqslant 20 \%$ with premenstrual deterioration, it was considered to comply with PMA criteria from the functional viewpoint (objective).

A database was designed which, after the inclusion of symptoms data and PF values for the days studied, informed us if the patient met PMA criteria for clinical or functional criteria or both. In the calculation of percentages, the constant 0.01 was added to the denominator for all cases in order to avoid denominators of zero that would block the calculation of variability.

The comparison of averages of each symptom (coughing, wheezing, tightness across the chest and breathlessness) in the two phases under analysis (preovulatory and premenstrual) was done via the paired t-test. The relationship between the categorical variables for having PMA and the classification of asthma according to GINA 2005 criteria (intermittent, mildly persistent, moderate or serious) was analysed by means of the Chi-squared test using the SPSS version 16 statistical package (SPSS Inc., Chicago, IL, USA).

\section{RESULTS}

Initially there were 141 asthmatics of fertile age. 25 patients did not complete the follow-up questionnaire and were excluded from the study. 221 questionnaires were obtained from 116 patients, with 105 completing the two menstrual cycles. This represented $82.26 \%$ (116 out of 141) of the asthmatics included at the start. 13 females had taken OC. These were later excluded from the analysis. The study was definitively carried out on 103 patients.

Table 1 shows the baseline characteristics of the patients, as well as the differences between those who had semi-objective PMA in the first cycle and those who did not.

Regarding the prevalence of PMA according to the definition applied, subjective perception of premenstrual deterioration was referred to by $43.7 \%$ of patients (95\% CI $34.4-53.4 \%$ ). In terms of the semi-objective evaluation of PMA, an exacerbation of symptoms in the first cycle was $44.7 \%$ (35.3-54.3\%). In one or other of the two cycles it registered $59.6 \%$ (49.4-69.1\%). As semi-objective deterioration of symptoms was required in both cycles, the frequency dropped to $22.3 \%$ (14.8-31.6\%). Three females $(2.9 \%$ ) presented functional (objective) criteria, all with a PF deterioration in the premenstrual phase of between $20 \%$ and $40 \%$ but none higher than $40 \%$. All three met semiobjective criteria, thus the PF measurement did not contribute any new cases.

The group that did not satisfy semi-objective PMA criteria in the first cycle presented two different types of behaviour: 31 females $(54.4 \%)$ showed no significant modification in symptoms between the preovulatory and premenstrual phases of the cycle, while the other $26(45.6 \%)$, contrary to that of the PMA group, showed considerable improvement $(>20 \%)$ in symptoms in the premenstrual phase. 
TABLE 1 Baseline characteristics of the patients

\begin{tabular}{|c|c|c|c|c|}
\hline & Global & Premenstrual asthma ${ }^{\#}$ & No premenstrual asthma ${ }^{\#}$ & p-value \\
\hline Age yrs & $28.6 \pm 8.8(14-47)$ & $26.9 \pm 6.5(16-37)$ & $28.78 \pm 9.93(14-47)$ & 0.41 \\
\hline FVC $\%$ & $93.69 \pm 13.66$ & $91.25 \pm 12.49$ & $95.22 \pm 14.31$ & 0.31 \\
\hline FEV $_{1} \%$ & $90.61 \pm 18.1$ & $88.2 \pm 15.9$ & $92.11 \pm 19.4$ & 0.45 \\
\hline Tiffenau & $79.98 \pm 11.6$ & $80.2 \pm 11.5$ & $79.8 \pm 11.9$ & 0.92 \\
\hline
\end{tabular}

Data are presented as mean \pm SD (range) or mean \pm SD, unless otherwise stated. FVC: forced vital capacity; FEV1: forced expiratory volume in $1 \mathrm{~s} .{ }^{\#}:$ semi-objective criteria in the first cycle.

TABLE 2 Preovulatory and premenstrual peak flow values, according to different premenstrual asthma (PMA) definitions

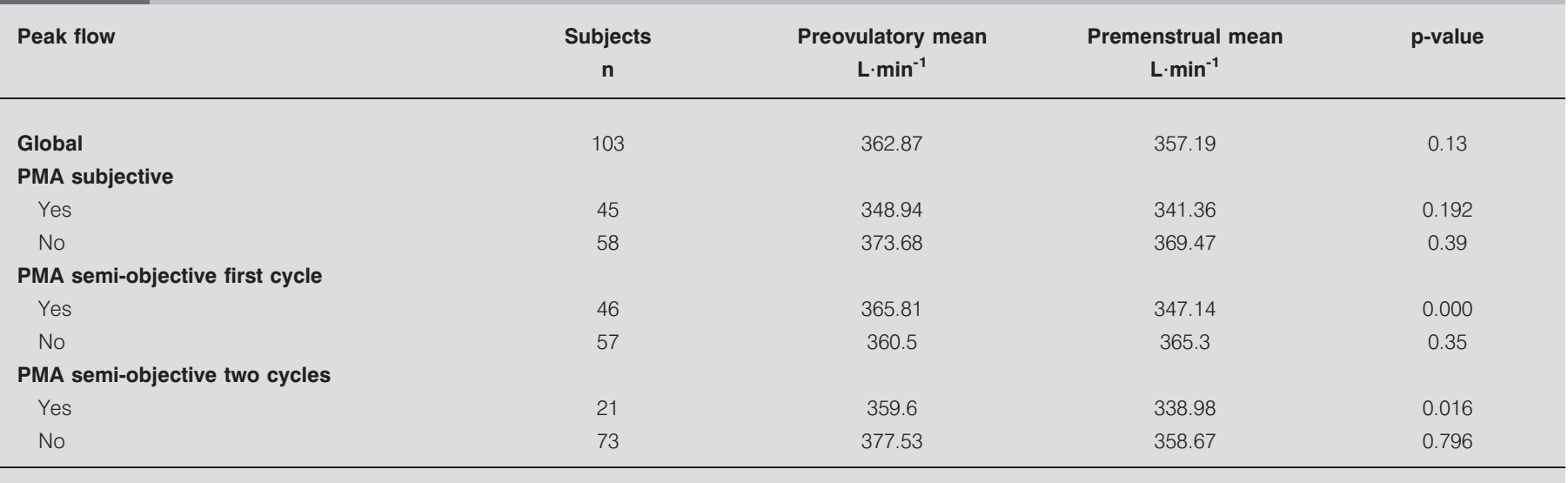

The PF in the preovulatory and premenstrual phase is shown in table 2. There is no change in PF with regard to the subjective definition. For the semi-objective definitions, there is a premenstrual fall in females with PMA.

Figures 1, 2 and 3 show the comparison between the average scores of daily symptoms [1] from the questionnaires in the two phases (preovulatory and premenstrual) in the patients with PMA (figs 1a, 2a and 3a) and those without PMA (figs 1b, $2 b$ and $3 b)$ according to the different definitions applied. In the PMA cases defined as subjective (fig. 1a and b), there were no notable differences between the preovulatory and premenstrual phases in the majority of symptoms. In patients with semi-objective PMA in the first cycle (fig. 2a), all their symptoms deteriorated significantly in the premenstrual phase. However, those who did not match these criteria saw their symptoms improve significantly in this phase, although the improvement was less relevant (fig. 2b). Females with PMA in both cycles (fig. 3a) also showed significant premenstrual deterioration, while those who did not fit the criteria showed no change (fig. 3b).

A total of $54.3 \%$ of the females who were semi-objective in their criteria in the first cycle also stated that their asthma worsened before menstruation (subjective criteria), versus 35.1\% of those who were not semi-objective $(p=0.05$; table 3$)$. With the requirement of semi-objective criteria in both cycles, the perception of premenstrual deterioration of their symptoms occurred with similar frequency $(47.6 \%$ versus $43.8 \%$; $\mathrm{p}=0.759)$.
Table 4 shows the classification of asthma severity in the 103 patients (39 mild intermittent, 19 mild persistent, 16 moderate persistent and 29 severe persistent) and the relationship between the severity of the asthma and having PMA according to the different definitions applied (subjective perception, or semi-objective criteria in one or two menstrual cycles).

PMA was frequent at all levels of asthma severity. Although the prevalence of PMA varied at the different levels of asthma severity for all definitions (table 4; raw Chi-squared p-value), no linear link was found between PMA prevalence and the severity of asthma (table 4; raw linear association p-value).

\section{DISCUSSION}

From the clinical viewpoint, PMA is defined as the exacerbation of asthmatic symptoms and/or PF values in some asthmatic females of fertile age in the luteal phase of the menstrual cycle or in the first days of menstruation.

From the epidemiological viewpoint, numerous studies have defined PMA as the patient's subjective appreciation of an exacerbation of their asthma in the premenstrual phase $[2,10$, 11]. In 1963, REES [10] reported that $37 \%$ of asthmatic females referred to a premenstrual deterioration of their asthmatic symptoms. Later, various authors referred to a PMA prevalence of between $20 \%$ and $40 \%$ in line with a "Yes" answer to "Does your asthma get worse before menstruation?" ELIASSON et al. [1] found 33\%, HANLEY [11] found 35\%, and 

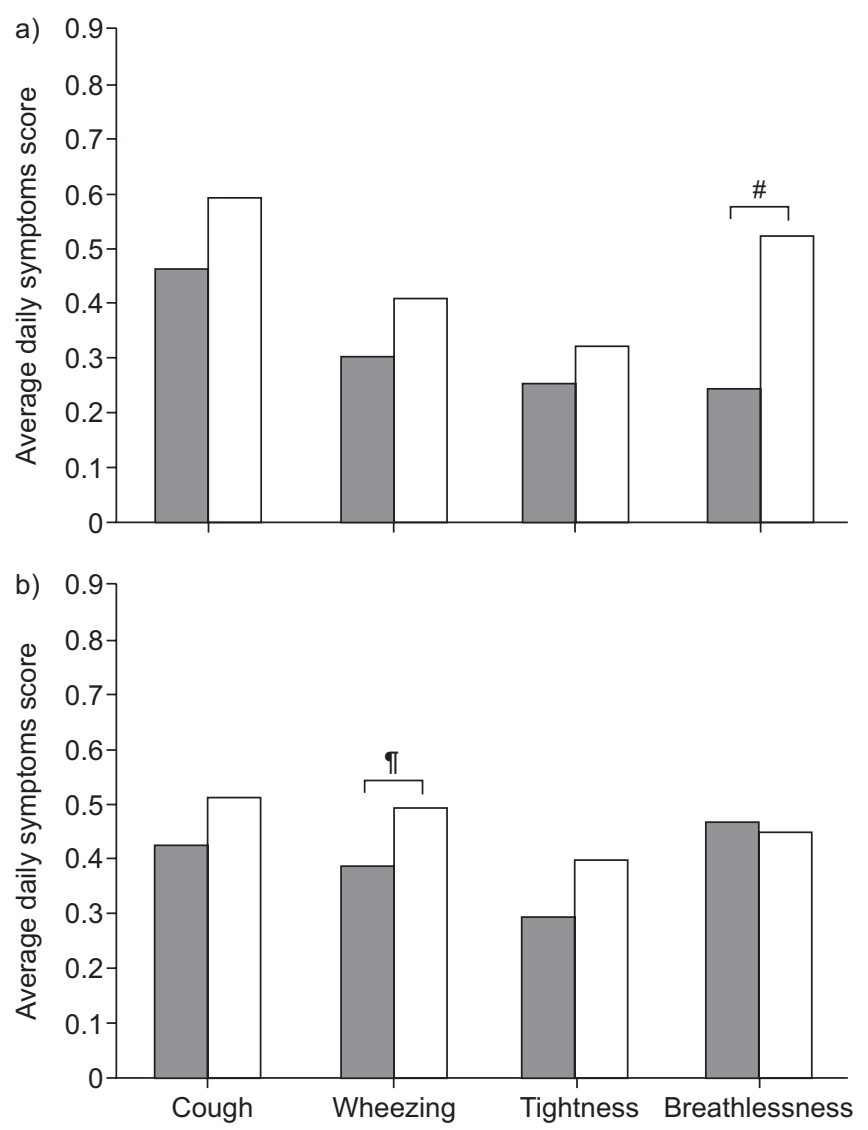

FIGURE 1. Comparison of the average daily symptoms score for each respiratory symptom collected for the two phases under analysis (preovulatory ( $\square$ ) and premenstrual $(\square)$ ), according to a) meeting or b) not meeting criteria for subjective premenstrual asthma. ${ }^{*}: p=0.001 ;{ }^{\circ}: p=0.024$.

GIBAS et al. [12] found 40\%. AgARWAL and SHAH [2] studied 100 asthmatic females of fertile age, finding that 23 acknowledged subjective premenstrual deterioration. Of these, $17(73.9 \%)$ felt worse the week before, and eight also felt worse during the week of menstruation. One patient felt worse 2 days before menstruation started. REES [10] reported that $90 \%$ got worse in the week prior to menstruation and $10 \%$ during the week of menstruation. We consider the 6-day premenstrual phase to be the 5 days prior to menstruation and the first day of bleeding.

In our study, we considered PMA from the subjective viewpoint when the patient indicated an exacerbation of her asthma in the premenstrual phase. The $43.7 \%$ figure is above that found in other published works.

Attempts to define PMA criteria objectively have been made since ELIASSON et al. [1] and ENSOM et al. [4]. These authors pose a methodology to try to define PMA with "objective" and standardised criteria that show a premenstrual deterioration of the asthmatic symptoms or PF values in relation to the preovulatory phase. The asthmatic symptoms are collected daily using a methodology that can compare and quantify the preovulatory and premenstrual stages. The data were collected by the patients themselves, which implied a certain subjective component. However, this clearly contributes to standardisation and a methodology in the collection of symptoms in order
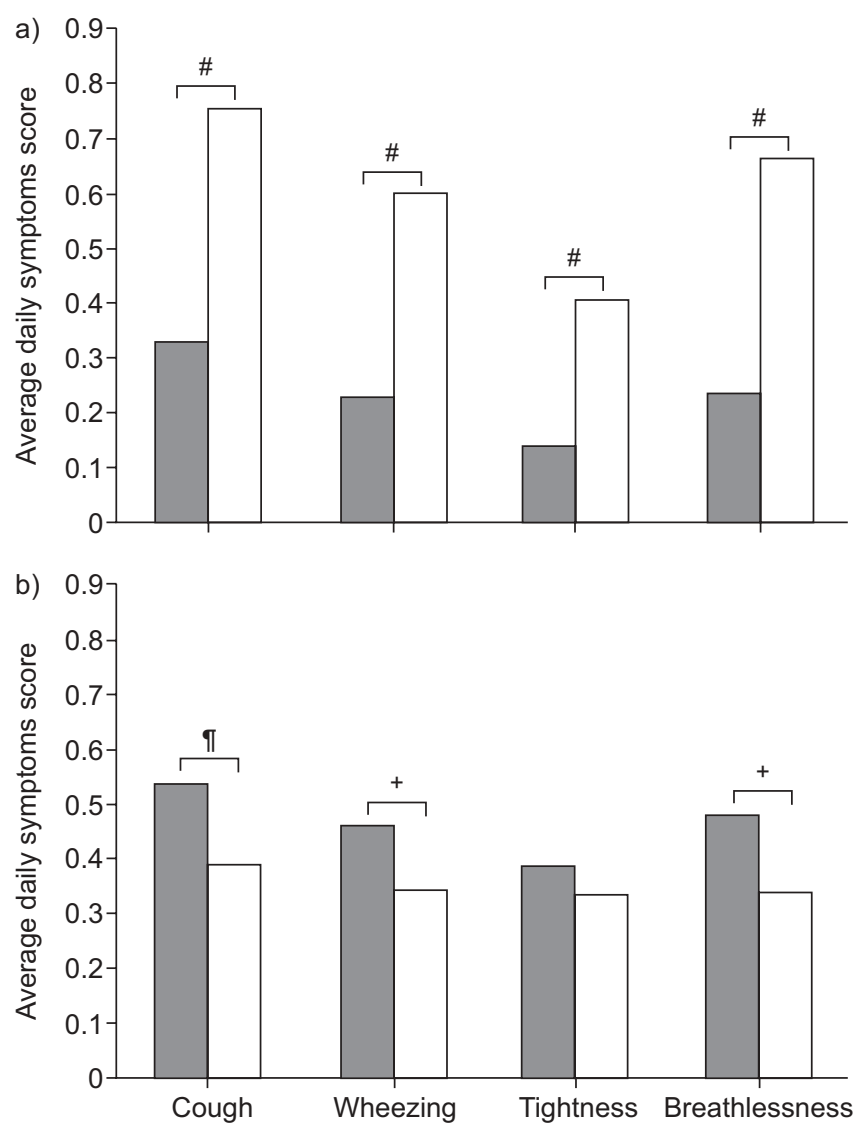

FIGURE 2. Comparison of the average daily symptoms score for each respiratory symptom collected for the two phases under analysis (preovulatory ( $\square$ ) and premenstrual $(\square)$ ), according to a) meeting or b) not meeting criteria for semiobjective premenstrual asthma in the first cycle. \#: $p=0.001 ; \boldsymbol{q}: p=0.023$; $+: p=0.004$.

to try to make the results objective. This enables later comparisons between studies to be far more objective than the simple answer to the question "Does your asthma get worse before menstruation?" This definition is applied in epidemiological studies and especially in clinical trials $[8,9]$, to compare the effect of various treatments on PMA.

In our study, we define semi-objective PMA as the premenstrual deterioration (compared with the preovulatory phase) of symptoms. We make the comparison by applying the methodology previously described, based on work by ELIASSON et al. [1] and ENSOM et al. [4].

We have analysed these semi-objective PMA criteria in two consecutive menstrual cycles, requiring conformity in one or both cycles. In this regard, authors such as ENSOM and coworkers $[4,9,13]$ and PASAOGLU et al. [8] believe the evaluation of only one menstrual cycle to be sufficient for cataloguing PMA and for designing a subsequent clinical trial. In addition, AGARWAL and SHAH [2] report that $>61 \%$ of patients with PMA show premenstrual deterioration in all cycles, $39 \%$ every two or three cycles and only one patient felt worse every three or four cycles. MURPHY and GIBSON [14] found that, of the four females studied over four consecutive cycles, two had PMA criteria, one in all four cycles and the other in three cycles. 

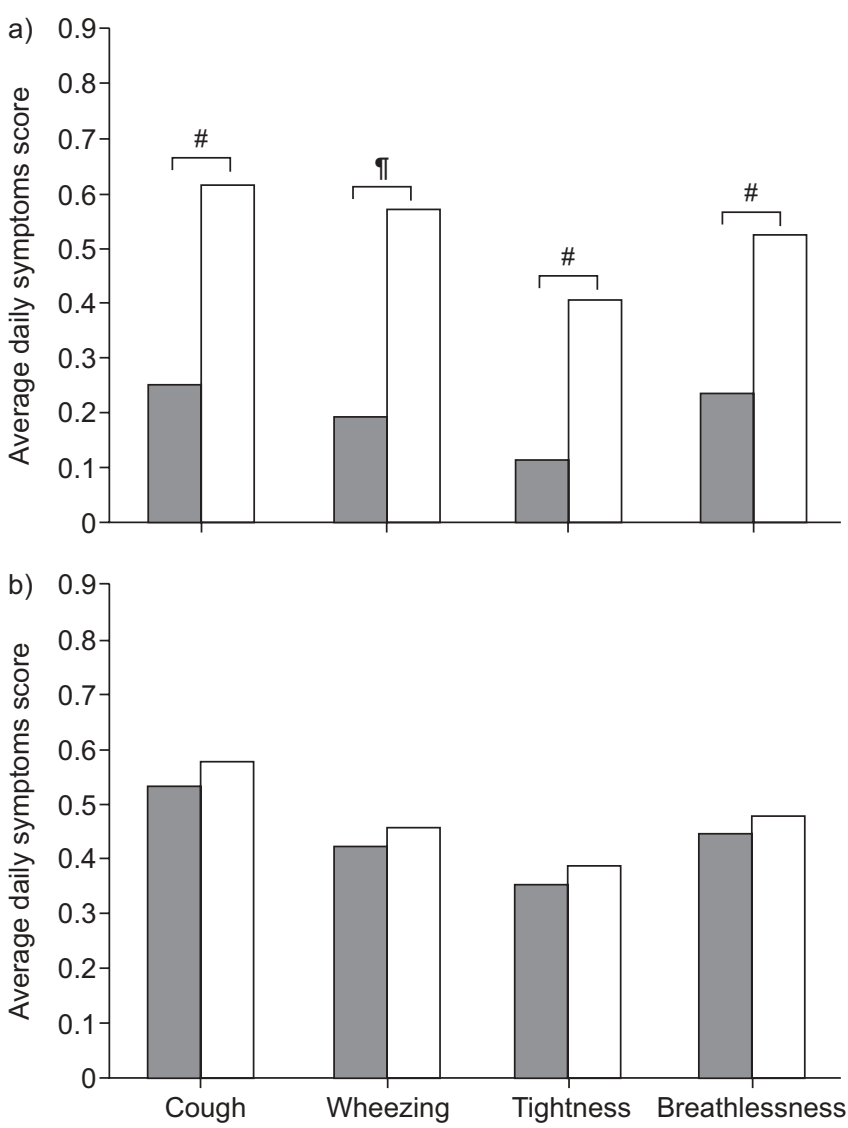

FIGURE 3. Comparison of the average daily symptoms score for each respiratory symptom collected for the two phases under analysis (preovulatory ( $\square$ ) and premenstrual $(\square)$ ), according to a) meeting or b) not meeting criteria for semiobjective premenstrual asthma in both menstrual cycles. ${ }^{*}: p=0.001 ;{ }^{\bullet}: p=0.003$.

Thus, if criteria are met in one isolated cycle, the majority of patients would be taken. Conversely, HALBREICH and ENDICOTT [5], on studying the methodology of the studies that analysed premenstrual changes, considered it compulsory to analyse two consecutive menstrual cycles.

We have found that the worsening of the score for symptoms (semi-objective criteria) in the first cycle was $44.7 \%$. It stood at $59.6 \%$ in either of the two menstrual cycles, slightly higher than those in the studies by ELIASSON et al. [1] (33\%) and ENSOM et al. [4]. The frequency fell to $22.3 \%$ if semi-objective symptom deterioration was required in both cycles.

The group that did not satisfy semi-objective PMA criteria in the first cycle presented two different types of behaviour: 31 females $(54.4 \%)$ showed no significant modification in symptoms between the preovulatory and premenstrual phases of the cycle, while the other $26(45.6 \%)$ behaved paradoxically and contrary to the PMA group, showing considerable improvement $(>20 \%)$ in symptoms in the premenstrual phase. A possible explanation for this behaviour is the definition of PMA itself, which excludes females whose symptoms do not worsen. Among these, one would expect to find females whose situation does not alter and others whose symptoms improve. The study of sexual hormone levels in the preovulatory and premenstrual stages might reveal data with respect to this.

\begin{tabular}{|c|c|c|c|}
\hline \multirow[t]{2}{*}{ TABLE 3} & \multicolumn{3}{|c|}{$\begin{array}{l}\text { Relationship between the diagnosis of semi- } \\
\text { objective premenstrual asthma (PMA) and } \\
\text { subjective PMA }\end{array}$} \\
\hline & & Subjective PMA & p-value \\
\hline \multicolumn{3}{|c|}{ Semi-objective PMA in cycle 1} & 0.05 \\
\hline Yes & & $54.35(25 / 46)$ & \\
\hline No & & $35.1(20 / 57)$ & \\
\hline \multicolumn{3}{|c|}{ Semi-objective PMA in cycles 1 and 2} & 0.759 \\
\hline Yes & & $47.6(10 / 21)$ & \\
\hline No & & $43.8(32 / 73)$ & \\
\hline
\end{tabular}

\begin{tabular}{|c|c|c|c|c|}
\hline \multirow[t]{3}{*}{ TABLE 4} & \multicolumn{4}{|c|}{$\begin{array}{l}\text { Relationship between the severity of asthma } \\
\text { and having premenstrual asthma (PMA) } \\
\text { according to different definitions }\end{array}$} \\
\hline & & \multirow{2}{*}{$\begin{array}{l}\text { Subjective } \\
\text { PMA }\end{array}$} & \multicolumn{2}{|c|}{ Semi-objective PMA } \\
\hline & & & Cycle 1 & Cycles 1 and 2 \\
\hline \multicolumn{5}{|c|}{ GINA severity } \\
\hline Mild interr & & $30.8(12 / 39)$ & $25.6(10 / 39)$ & $15.2(5 / 33)$ \\
\hline Mild persi & & $52.6(10 / 19)$ & $78.9(15 / 19)$ & $47.1(8 / 17)$ \\
\hline Moderate & rsistent & $75(12 / 16)$ & $50(8 / 16)$ & $25(4 / 16)$ \\
\hline Severe pe & stent & $37.9(11 / 29)$ & $44.8(13 / 29)$ & $15.6(5 / 32)$ \\
\hline Total & & $43.7(45 / 103)$ & $44.7(46 / 103)$ & $22.3(21 / 94)$ \\
\hline Chi-squar & $p$-value & 0.018 & 0.002 & 0.044 \\
\hline Linear ass & iation $\mathrm{p}$-value & 0.323 & 0.184 & 0.734 \\
\hline \multicolumn{5}{|c|}{$\begin{array}{l}\text { Data are presented as } \%(n / n) \text {, unless otherwise stated. }{ }^{\#} \text { : Global Initiative for } \\
\text { Asthma (GINA) } 2005 \text { classification [7]. }\end{array}$} \\
\hline
\end{tabular}

The analysis of the variations in lung function during the menstrual cycle by means of PF has been studied by various authors $[15,16]$ and can be considered objective criteria. AgARWAL and SHAH [2] found a link between the symptoms registered in the questionnaire and the PF values, indicating that the deterioration in asthma in the perimenstrual phase is due to an increase in resistance in the air passage and not just to an increase in the perception of the symptoms. Other studies reveal patients who suffer a perimenstrual deterioration of $\mathrm{PF}$ values without any deterioration in their symptoms. It seems that PF variations in relation to the menstrual cycle are minimal in healthy nonasthmatic females [3]. In future studies, we will be able to take objective measurements of asthma variation throughout the menstrual cycle, specifically inflammation, by measuring nitric oxide (NO) in exhaled air. Recently, FARHA et al. [17] indicated that asthmatic females "experience cyclic changes in airflow as well as gas transfer and membrane diffusing capacity supportive of a hormonal effect on lung function". Airflow and lung diffusing capacity varied over the menstrual cycle, with peak levels during menses that subsequently declined to nadir in the early luteal phase. 
Regarding objective PMA criteria and their impact on prevalence, MURPHY and GIBSON [14] only consider the analysis of PF variations to be objective. In our study, we define objective PMA as the premenstrual deterioration (compared to the preovulatory phase) of PF values. We found a variation of $>20 \%$ in $2.9 \%$ of patients and the PF measurement did not contribute new cases.

As far as we know, there is only one previous study, by MURPHY and GIBSON [14], that presents the variation of PMA prevalence according to the definition applied. In that study, carried out on 28 asthmatic females, $43 \%$ (13 out of 28 ) of patients had subjective PMA; $86 \%$ (24 out of 28) showed changes in symptoms (cough, wheeze eye irritation and nose irritation) and 14\% (4 out of 28) showed PF variations. These data, like those obtained in our analysis, clearly reveal the variation in PMA frequency according to the definition applied and the need to define it clearly in order to be able to compare study results.

Our aim has not been to find the best PMA definition. Figures 2 and 3 show that the semi-objective definitions detect bigger differences between the preovulatory and premenstrual phases in asthmatic females. We believe it is too stringent to require semi-objective criteria in two consecutive cycles (it contributes a prevalence of 22.3\%). Our results lead us to think that the definition by semi-objective criteria in a menstrual cycle is what best defines the problem of PMA. Objective criteria, which demands a PF variation of $>20 \%$, is much more restrictive (contributing a prevalence of $2.9 \%$ ). Perhaps other objective criteria such as variations during the NO cycle, together with a longer register of cycles, would allow us to obtain a more valid PMA definition based on the presence of criteria in a greater or lesser number of cycles.

We have excluded from our study the 13 females who took OC, because of the influence of the drug on hormonal levels, the main aetiopathogenic factor related to PMA [5]. Of the 13 patients who took OC, six were semi-objective for PMA in the first cycle. These PMA prevalence data are similar to those of patients who did not take OC, and suggest that taking OC is not linked to the presence of PMA. This is an interesting development for future research.

Our study has tried to relate the subjective and semi-objective PMA criteria analysed. The data in table 3 suggest that there exists a relationship between subjective and semi-objective criteria, particularly when evaluating a menstrual cycle, thus we must question all asthmatics of fertile age about the possibility of subjective premenstrual deterioration.

We have also analysed the relationship between the severity of asthma and the different definitions (table 4). Classically, PMA is seen as a factor possibly related to "difficult asthma", to certain serious asthma crises [18] and to an increase in hospitalisations during the perimenstrual phase [1, 19]. SUZUKI et al. [20] report that their 54 patients with PMA had asthma that was more serious, unstable and hard to control (requiring oral corticoids), with more frequent visits to the emergency department and a higher intolerance of aspirin ( $25.5 \%$ versus $8.4 \%)$.
We also analyse a link between PMA and asthma severity. PMA was distributed irregularly among the different groups of GINA severity according to all definitions. We found PMA at all levels of asthma severity, with mild persistent being the most prevalent for PMA according to the semi-objective definitions, and moderate persistent showing greatest prevalence for the subjective definition. Again, we find arguments to justify the investigation of possible PMA in all the degrees of asthma severity.

We conclude that the prevalence of PMA in our working environment is high, similar to that reported in the literature, although it varies significantly according to the definition applied. Asthmatic females with PMA viewed the objective deterioration of their symptoms subjectively. Among those patients who did not meet semi-objective PMA criteria, there was an important subgroup that presented inverse clinical behaviour, with improvements in premenstrual symptoms. PMA is present at all levels of asthma severity but with no clear link to that severity.

The high prevalence of PMA, the subjective perception of this deterioration and its presence at all levels of asthma severity lead us to urge research into possible premenstrual deterioration in all asthmatic females of fertile age.

\section{SUPPORT STATEMENT}

This study was financed in part by grants from the Asociación de neumólogos del sur (Neumosur) in 2003 and the Health Ministry of the Regional Autonomous Government of Andalusia in 2005 (both Seville, Spain).

\section{STATEMENT OF INTEREST}

None declared.

\section{ACKNOWLEDGEMENTS}

We wish to thank the following medical personnel for their help in performing the field study: E. Maldonado and J.A. Bernal Rodríguez (Hospital Juan Ramón Jiménez, Huelva, Spain), M.J. Chocrón Giráldez and M. Pinto Tenorio (Hospital Comarcal de la Serranía de Ronda, Málaga, Spain), P. Calvo Tudela (Hospital de Baza, Granada, Spain) and P. Pérez Navarro (Hospital Vírgen del Rocío, Seville, Spain).

\section{REFERENCES}

1 Eliasson O, Scherzer HH, DeGraff AC Jr. Morbidity in asthma in relation to the menstrual cycle. J Allergy Clin Immunol 1986; 77: 87-94.

2 Agarwal AK, Shah A. Menstrual-linked asthma. J Asthma 1997; 34: 539-545.

3 Chong E, Ensom MH. Peak expiratory flow rate and premenstrual symptoms in healthy nonasthmatic women. Pharmacotherapy 2000; 20: 1409-1416.

4 Ensom MH, Chong E, Carter D. Premenstrual symptoms in women with premenstrual asthma. Pharmacotherapy 1999; 19: 374-382.

5 Halbreich U, Endicott J. Methodological issues in studies of premenstrual changes. Psychoneuroendocrinology 1985; 10: 15-32.

6 Tan KS. Premenstrual asthma: epidemiology, pathogenesis and treatment. Drugs 2001; 61: 2079-2086.

7 Global Initiative for Asthma. Global Strategy for Asthma. Management and Prevention NHLBI/WHO Workshop Report. 2005. Available from http://www.ginasthma.com. 
8 Pasaoglu G, Mungan D, Abadoglu O, et al. Leukotriene receptor antagonists: a good choice in the treatment of premenstrual asthma? J Asthma 2008; 45: 95-99.

9 Ensom MH, Chong G, Zhou D, et al. Estradiol in premenstrual asthma. A double-blind, randomized, placebo-controlled, crossover study. Pharmacotherapy 2003; 23: 561-571.

10 Rees L. An aetiological study of premenstrual asthma. J Psychosom Res 1963; 7: 191-197.

11 Hanley SP. Asthma variation with menstruation. $\mathrm{Br} J$ Dis Chest 1981; 75: 306-308.

12 Gibas CJ, Coutts II, Lock R, et al. Premenstrual exacerbation of asthma. Thorax 1984; 39: 833-836.

13 Ensom MH. Gender-based differences and menstrual cycle-related changes in specific diseases: implications for pharmacotherapy. Pharmacotherapy 2000; 20: 523-539.

14 Murphy VE, Gibson PG. Premenstrual asthma: prevalence, cycleto-cycle variability and relationship to oral contraceptive use and menstrual symptoms. J Asthma 2008; 45: 696-704.
15 Nakasato H, Ohrui T, Sekizawa K, et al. Prevention of severe premenstrual asthma attacks by leukotriene receptor antagonists. J Allergy Clin Inmunol 1999; 104: 585-588.

16 Magadle R, Berar-Yanay N, Weiner P. Long-acting bronchodilator without premenstrual exacerbation of asthma. Respir Med 2001; 95 : 740-743.

17 Farha S, Asosingh K, Laskowski D, et al. Effects of the menstrual cycle on lung function variables in women with asthma. Am J Respir Crit Care Med 2009; 180: 304-310.

18 Martínez Moragón E, Plaza V, Serrano J, et al. Near-fatal asthma related to menstruation. J Allergy Clin Immunol 2004; 113: 242-244.

19 Skobeloff EM, Spivey WH, Silverman R, et al. The effect of the menstrual cycle on asthma presentations in the emergency department. Arch Intern Med 1996; 156: 1837-1840.

20 Suzuki K, Hasegawa T, Koya T, et al. Analysis of perimenstrual asthma based on questionnaire surveys in Japan. Allergol Int 2007; 56: $249-255$. 